EVS26

Los Angeles, California, May 6-9, 2012

\title{
Grid integration of electric vehicles considering the mobility needs
}

\author{
Markus Litzlbauer ${ }^{1}$ \\ ${ }^{1}$ Institute of Energy Systems and Electrical Drives, Vienna University of Technology, \\ Gusshausstr. 25 /E370-1, 1040 Vienna, Austria, Email: markus.litzlbauer@tuwien.ac.at
}

\begin{abstract} engineering, among others, the following questions arise:

- Where should be charging infrastructure for electric vehicles created?

- Which connection power should be provided?

- How big is the additional grid load?

- What must be done to prevent overloading?
\end{abstract}

Battery electric vehicles combined with renewable energies have a huge potential to reduce $\mathrm{CO} 2$ emissions, especially in the field of motorized individual transport. However, a future change from combustion engines to electric drives leads to new problems and challenges. From the perspective of energy

These questions are dealt with in the Austrian research projects "Smart Electric Mobility" and "V2G Strategies" with national partners from academia and industry (funded by the Austrian Climate and Energy Fund, programme "New Energy 2020"). The methods and selected results are presented in this paper.

Keywords: BEV (battery electric vehicle), load management, passenger car, photovoltaic, simulation

\section{Introduction}

The path to a sustainable and environmentally friendly mobility in the motorized individual transport will bring an increasing electrification of the drive train. In recent years, the battery characteristics have significantly improved. However, the achievable energy densities allow no ranges comparable to conventional vehicles. Therefore, for pure electric vehicles (EV) a charging infrastructure is necessary, which relies on an entirely different principle.

Electric vehicles normally will be charged at locations where they are parked, but not during an additional interruption along a trip. This will be only in exceptional cases the perfect solution for the EV.

The correct dimensioning of the charging infrastructure is based on the energy demand (proportional to the distances travelled) and the parking locations of the vehicles (with possibility to charge). This change also means that EV will be charged not only at critical points in public spaces, but to a large extent in private parking spaces and garages. This leads to a significant additional load for the distribution grid.

In this paper the methods and selected results produced by the Vienna University of Technology are discussed. 


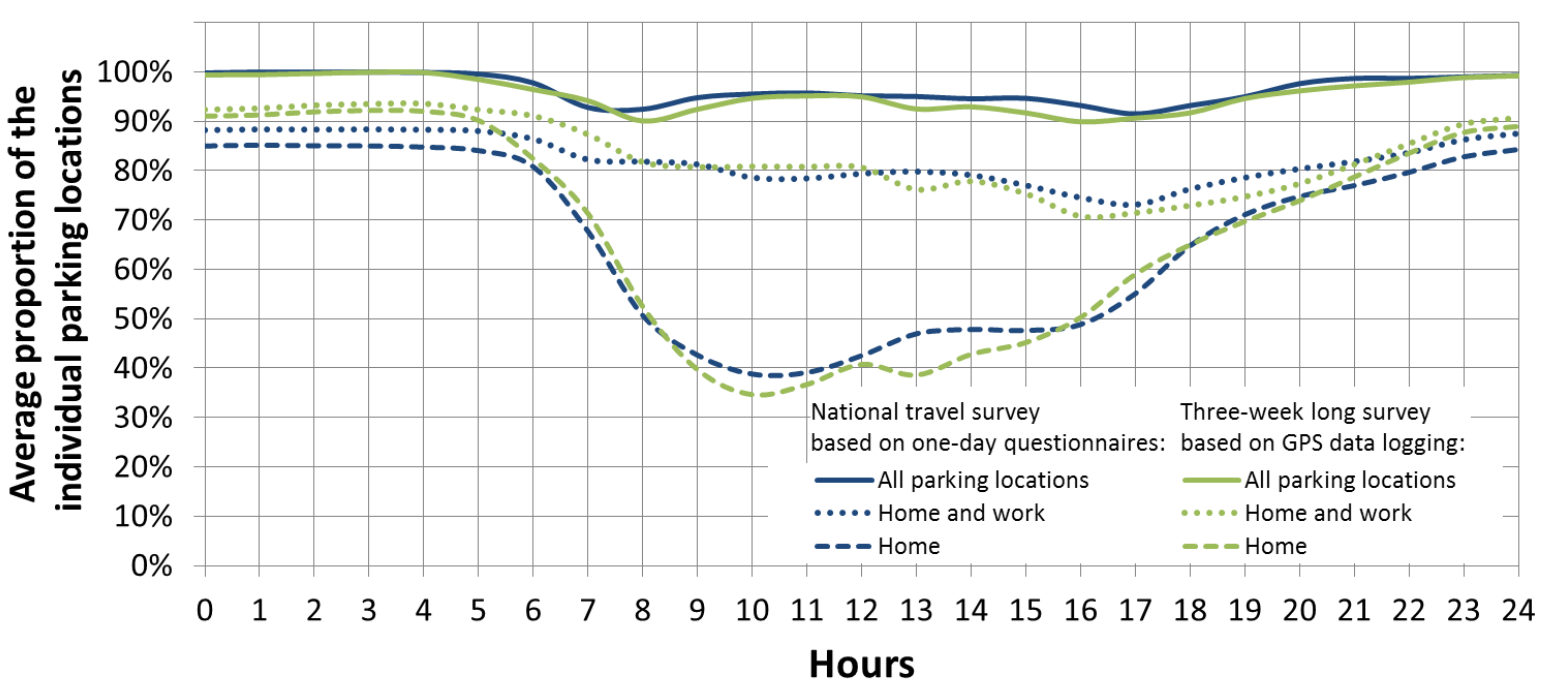

Figure 1: Time course of the average proportion of the individual parking locations

\section{Travel survey data}

As data basis for the following energy-related considerations two different data sets of the motorized individual transport in Austria are used.

The first, a survey based on GPS data logging, were designed and executed by the University of Natural Resources and Life Sciences Vienna. For this long-term survey (LTS) they have installed GPS logging devices in gasoline-powered vehicles of about 30 individual subjects. The continuous trip recording was carried out in Vienna and its surroundings for a period of more than three weeks per private car in the year 2010 [1].

The second one is a national travel survey (NTS) based on one-day questionnaires. It was executed in Salzburg in the year 2004. Over 3400 private drivers were interviewed but only trips on weekdays (Mon-Fri) were considered [2].

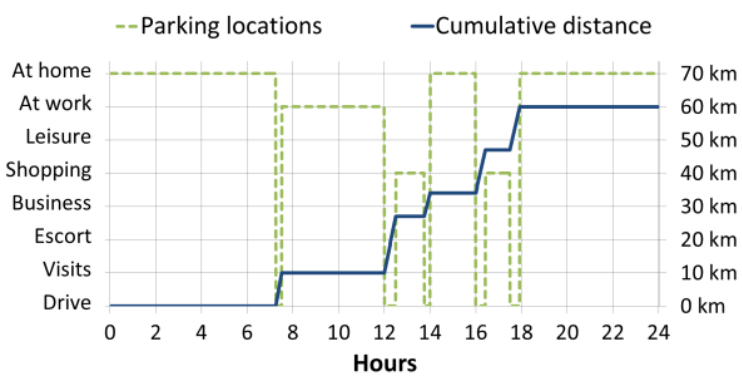

Figure 2: Time course of parking locations and cumulative distance (one exemplary vehicle)

For each trip of both surveys the parameters "start and end time", "duration", "distance" and "trip purpose" are extracted. Using the parameters, the driving profiles and parking profiles for each vehicle are created (see Figure 2). It is assumed that no vehicle is shared with another person.

The analysis of the traffic data (weekdays: Monday to Friday) for both surveys shows very similar results, despite different numbers of vehicles and different periods under observation. In Figure 1 the time course of parking locations for both surveys can be seen ${ }^{1}$. More than $85 \%$ of all vehicles park at home in the night between 00:00 and 04:00 a.m. and at least $35 \%$ at all times of the day. Further analyses reveal that the share of vehicles parked at work has its maximum at 10:00 a.m. and is about $46 \%$. The next common parking locations are "Leisure" and "Visiting friends". However, they never achieve individually a proportion over $7 \%$.

The results show that the vehicles principally park at the locations "at home" and "at work". Therefore, charging infrastructure should be primarily built at these two parking locations.

\section{Vehicle-related modelling and simulation}

To determine the energy requirements of the synthesized electric vehicles a physical respectively numerical simulation has been created by the Austrian Institute of Technology ([1], [3]). The model was developed in DYMOLA and it is based on the MODELICA Standard Library.

\footnotetext{
${ }^{1}$ It was assumed that each day around $17 \%$ of all vehicles didn't make a single trip. This should consider the "non-mobile persons" and was calculated from the LTS.
} 
In combination with the traffic data of the LTS they could calculate the specific consumption of an adequate electric vehicle. These results are also used for the continuing analyses of the NTS.

Based on the findings, in relation to parking location and energy demand of electric vehicles, in both research projects several modular MATLAB programs were developed. Those allow executing comprehensive energy-related analyses for both types of surveys. At the level of one battery electric vehicle (BEV) it is possible to generate the temporal profiles of the travelled distances, the parking locations, the state of charge (SOC) and the required load. Therefor the battery capacity, the relevant parameters of the charging characteristics, the expansion level of the charging infrastructure, the load power, the specific consumption, various losses and efficiencies, as well as several variants of consumption- and production-oriented charging strategies can be freely chosen and simulated. In the Following the modular programs will be referred to as MATLAB-Tool.

\section{Feasibility analysis}

The given charging infrastructure and battery capacity of an electric vehicle restrict the possible distance of the entire trip chain ${ }^{2}$.

Only trip chains which can be passed with a BEV - under the given Framework - are used in the following analyses. That means that the state of charge (SOC) always has to be above the minimum limit.

In the first step using the MATLAB-Tool a sensitivity analysis including the parameters "battery capacity" and "charging power" was carried out. The results of the sensitivity analysis of both surveys are shown in Figure 3. The small marker points illustrate the findings of the individual variations, the major one the arithmetic mean. The influence of a parameter is given on the one hand by the course of the mean values and on the other by the dispersion of the individual variations.

The number of vehicles, which can be operated as electric vehicles, increase with growing of the parameter "battery capacity". The battery size

\footnotetext{
2 A "car trip" is defined as a single, one-way trip. Several successive car trips combined together are called trip chain.
}

clearly has the strongest influence on the satisfaction of the mobility needs, which is evident by the low dispersion of the individual variations. It was assumed that charging is only possible at the defined parking locations and interrupting a trip for recharge is not allowed.

However, the parameter "charging power" is in the middle constant for all three power values. In addition, the dispersion of the individual variations is relatively large and for this reason the charging power has a small influence on the satisfaction of the mobility needs.

As a result of the sensitivity analysis should be noted that a maximum connection power of $3.7 \mathrm{~kW}(\sim 230 \mathrm{~V}, 16 \mathrm{~A}$, single phase, hereafter called "normal charge") is sufficient during recharging. Charging powers to $22 \mathrm{~kW}(\sim 230 \mathrm{~V}$, $32 \mathrm{~A}$, three-phase) bring no additional benefit with respect to the satisfaction of the mobility needs. Higher connection powers (>100 kW) are only of interest, if trips are interrupted for quick charging.

$$
\begin{aligned}
& \triangle \text { Three-week long survey based on GPS data logging } \\
& \text { National travel survey based on one-day questionnaires }
\end{aligned}
$$
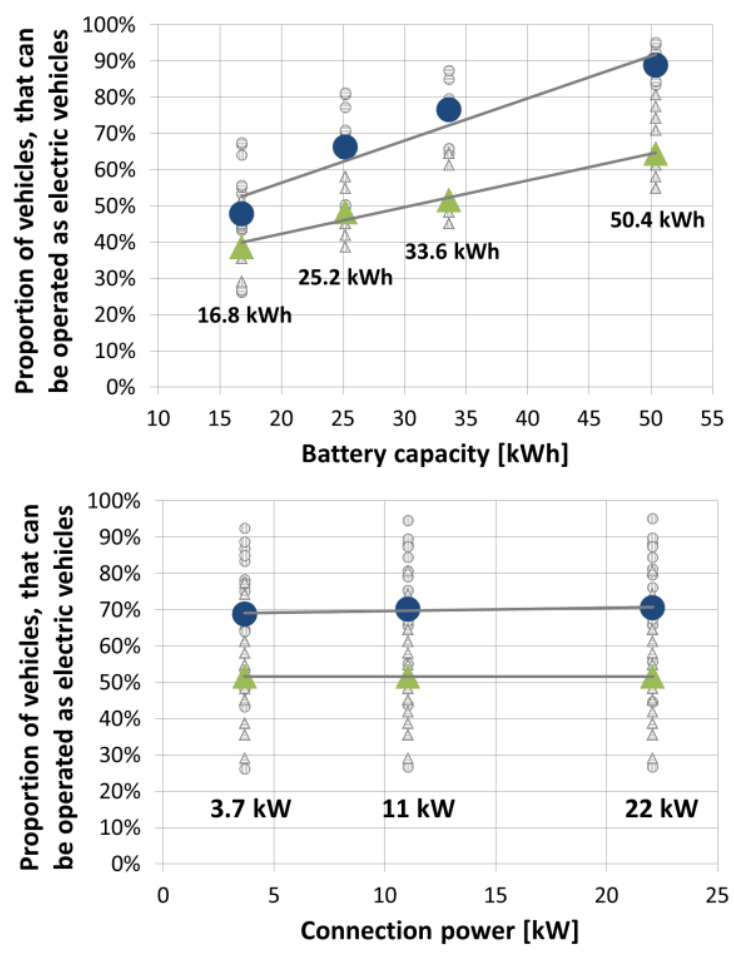

Figure 3: Sensitivity analysis for the parameters "battery capacity" (above) and "charging power" (below)

In the LTS each vehicle was recorded at least three weeks. However, the NTS only consists of one-day data. Therefore, the individual daily data were 
randomly combined to produce continuously 7 day data chains (existing of several working days). In the next analysis each vehicle was consistently simulated for the entire observation period.

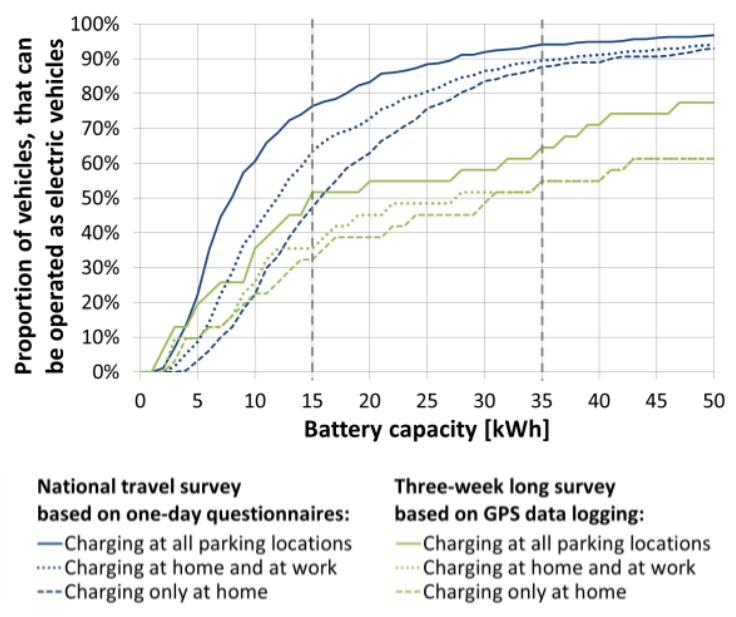

Figure 4: Possible electric vehicle usage in relation to the battery capacity

Figure 4 represents the results of this "Feasibility analysis" and shows the possible electric vehicle usage in relation to the battery capacity for different charging infrastructure levels of both surveys. Charging is only possible at considered parking locations and with a connection power of $3.7 \mathrm{~kW}$.

It is intended to show only tendencies with the battery capacity range of 0 to $50 \mathrm{kWh}$. Today's battery capacities are rather in the range of 15 to $35 \mathrm{kWh}$ (limited by two vertical dashed lines). The difference in the blue and green curves can be largely explained by the consideration of large trips in the LTS for "Visiting friends" at weekend - in contrast to the NTS.

\section{Uncontrolled charging profiles}

In this paper the term "uncontrolled charging" means, that the charging of the vehicle battery starts immediately after reaching a defined location equipped with a charging infrastructure. If there is only at home the possibility to charge the BEV, it represents the "worst case". This case plays a major role with respect to the electric grid load and so it will be discussed below.

Figure 6 shows the time courses of the SOC, the parking locations and the load profile of an exemplary BEV. The curves were obtained using the aforementioned MATLAB tool, and it was assumed that the Li-Ion battery has a capacity of $25 \mathrm{kWh}$, which is operated within the SOC limits of 10 to $90 \%$. The connecting power is $3.7 \mathrm{~kW}$ $(\sim 230 \mathrm{~V}, 16 \mathrm{~A}$, single-phase) and the battery charger has a UIa characteristics implemented. Charging infrastructure is only offered at home.

To estimate the load of a power distribution grid, primarily the sum of the load profiles of the individual $\mathrm{BEV}$ is of interest. The normalized sum is diagrammed in Figure 5 for both surveys. There are only those vehicles considered with their mobility needs met, which can be operated as BEV. The comparison of the normalized total charging profiles shows equality in the peak load. They occur between 18:00 and 18:30 o'clock and reach a peak of around $0.55 \mathrm{~kW}$ per BEV. Differences in the courses are due to local conditions and due to

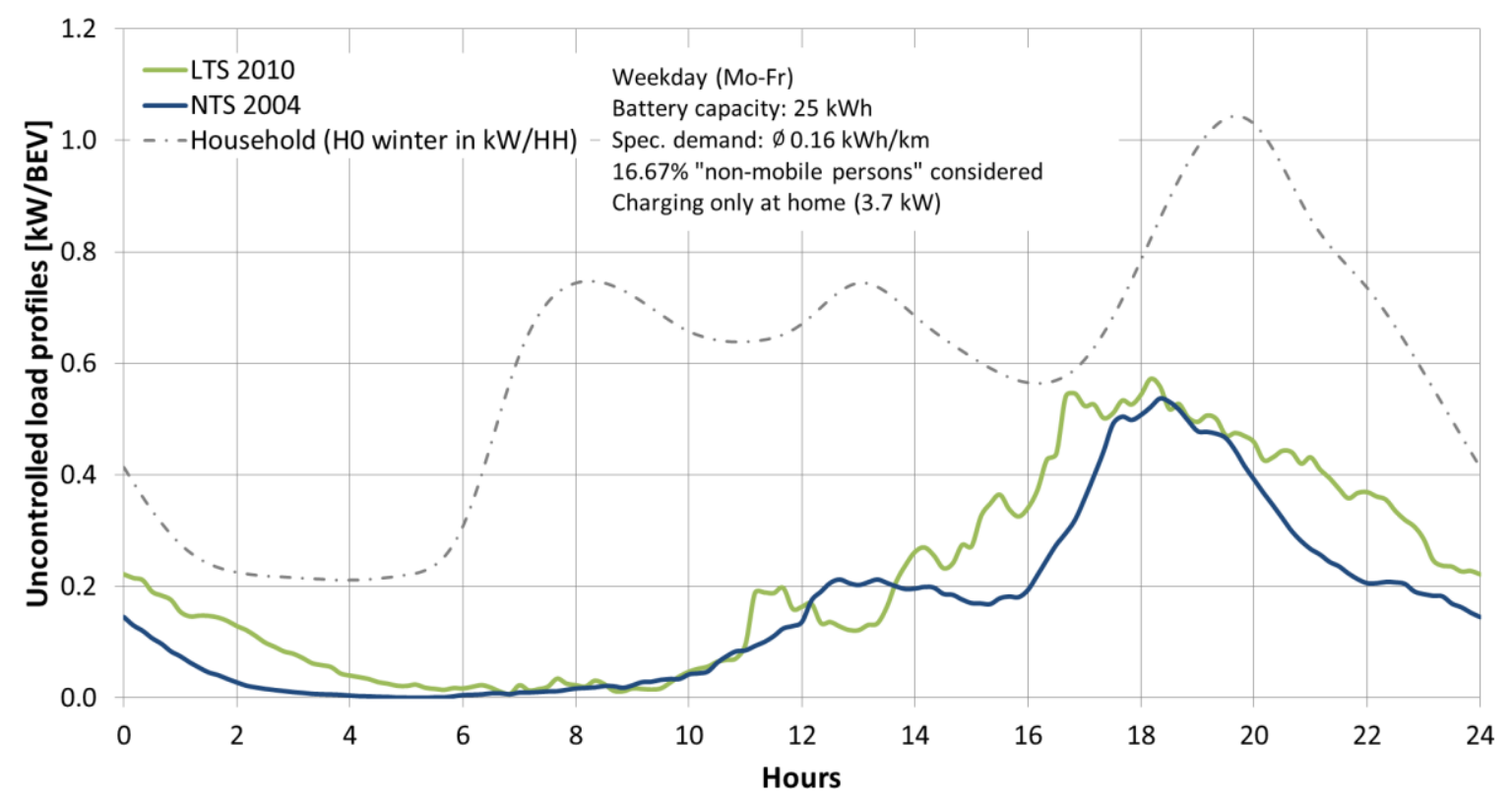

Figure 5: Normalized total load profiles of both surveys 
the different survey types.

Also significant is the comparison with the standardized household load profile. As shown in Figure 5, the average peak load of uncontrolled loading of a vehicle $(3.7 \mathrm{~kW})$ is more than the half of the average peak load of the H0-profile and they occur nearly at the same time. This case would lead to a significant increase of peak load in the distribution grid in evening.

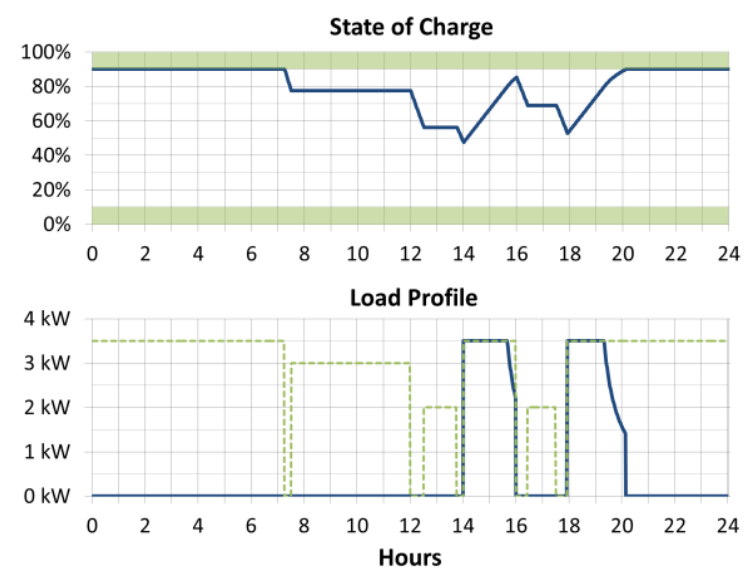

Figure 6: Time course of SOC and load profile for one exemplary electric vehicle

\section{$6 \quad$ PV-based charging strategy}

To reduce the load peak of uncontrolled charging in the evening, charging infrastructure can be expanded or controlled charging can be used. This chapter examines in this regard a PV-based charging strategy.
In the field of electric vehicles, the choice of the energy source plays an important role. This is because, if vehicles should cause less $\mathrm{CO} 2$ emissions than conventional fossil-driven vehicles, preferably renewable resources (such as solar or wind) must be used.

In this specific case it is tried to cover the charging demand - locally and at the same time - by using photovoltaic. For the analyses of this case, averaged minute values of a real solar power system are used.

The parameter "total coverage" is set to one, which means that the generation of the solar power systems per year is equal to the energy demand per year of the BEV.

For the modelling only the NTS data are used and averaged $1.9 \mathrm{kWp} \mathrm{/} \mathrm{BEV} \mathrm{of} \mathrm{solar} \mathrm{panels} \mathrm{are}$ installed at the parking location "at home". The average solar power generation is annually $1045 \mathrm{kWh} / \mathrm{kWp}$.

The realization of the model was carried out in MATLAB and aims to use as much energy as possible directly from photovoltaic. For this, the charging must be moved from the evening hours into the day. The charging control is dependent on the SOC, and is optimally adjusted for each individual vehicle. The following results were calculated assuming an "individual trip forecast" and only a constant-current charging.

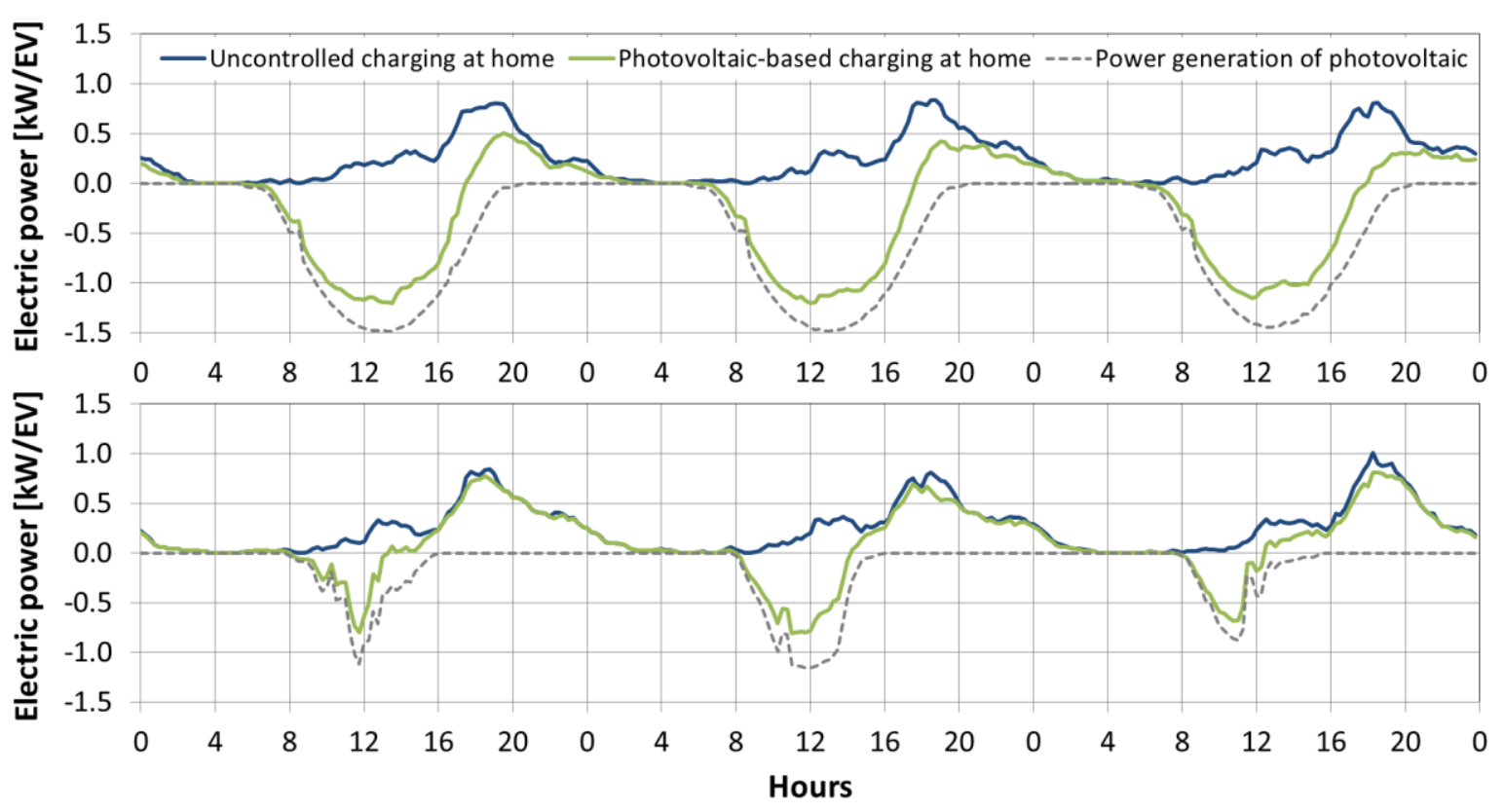

Figure 7: Average charging profiles for uncontrolled and PV-based charging at home (above: three days in July, below: three days in December) 
Figure 7 shows the optimized charging profile with PV usage for three exemplary days in June and December. For comparison, the uncontrolled load profile and the solar power profile is also diagrammed. It can be clearly seen that, on the one hand, a reduction of the grid load is possible in the summer through the direct PV usage. On the other hand, in winter almost the entire BEV energy must be provided by the distribution grid. To reduce the peak load also in winter, e.g. the power demand has to be shifted into the night using controlled charging.

Viewed over the entire year, a self coverage of up to $25.4 \%$ can be achieved in this case. The self coverage is the ratio of the BEV energy demand, which can be met directly by photovoltaic. An additional solar power system at the workplace may lead to an improvement of the performance issues.

At this point it should be noted that the underlying solar power supply principle has a positive effect on the voltage stability in the distribution grid. However, excessive solar power supplies can cause overvoltage and the use of singlephase inverters generates unbalanced loads.

\section{Summary}

At the Austrian research projects "Smart Electric Mobility" and "V2G Strategies" a three-weeklong GPS-based survey was generated and a national travel survey based on one-day questionnaires was used. Both are in the range of motorized individual transport in Austria. Based on these surveys, on the one hand, valuable information about travel behaviour and the satisfaction of the mobility needs are given and, on the other hand, important decisions for uncontrolled and PV-based charging of electric vehicles are attained.

The sensitivity analyses of the parameters "battery capacity", "charging power" and "charging infrastructure" and its follow-up feasibility analysis results in the future charging infrastructure needs. For the most vehicles a charging infrastructure "at home" and "at work" is adequate and connection powers above $3.7 \mathrm{~kW}$ are not necessary. However, the most important criterion for the satisfaction of the mobility needs is the battery capacity.

For uncontrolled charging at home with $3.7 \mathrm{~kW}$ the additional grid load is about $0.55 \mathrm{~kW}$ per $\mathrm{BEV}$. To prevent overloading in the distribution grid, charging infrastructure can be expanded or controlled charging can be used. In this paper a $\mathrm{PV}$-oriented charging strategy is introduced.

If a solar power system is installed at the parking location "at home" and the photovoltaic is directly used for charging the electric vehicles, coverage rates of about $25 \%$ can be achieved under the given framework - without compromising the everyday mobility needs.

\section{References}

[1] Leitinger, C., Litzlbauer, M., Schuster, A., et al. (2011, Vienna University of Technology): Smart Electric Mobility - Storage of Electric Car Batteries for Mobility Use and Grid Stability, FFG research project, NE2020, project number: 821886, Final Report, Vienna 2011.

[2] Herry M., Russ M., Steinacher I., Tinnauer C. Snizek S. (2005, urban administration of Salzburg): Mobilitätsanalyse Stadt Salzburg und Umgebung 2004, Final Report, Vienna, July 2005.

[3] Litzlbauer M. (2010, Vienna University of Technology): Generation of stochastic load profiles for mobile energy storages, EVS 25, China, November 2010, "EVS 25 Sustainable Mobility Revolution", (2010), S. 417 - 420.

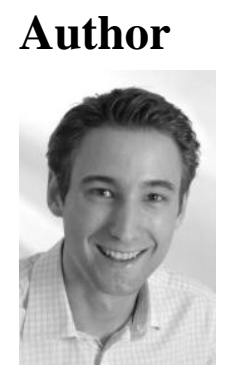

Markus Litzlbauer, MSc Gusshausstr. 25 / E370-1, 1040 Vienna, Austria Tel: +43158801370132 Fax: +43158801370199 markus.litzlbauer@tuwien.ac.at http://www.ea.tuwien.ac.at

He was born on October 24, 1981 in Austria. He studied Electrical Engineering in the branch of study Energy Engineering at the Vienna University of Technology and wrote his diploma thesis to the topic ,Modelling of stochastic load profiles of mobile energy stores". Since November 2009 he is active as a project assistant for research at the Institute of Energy Systems and Electrical Drives at the Vienna University of Technology in the range of grid integration of electric vehicles. 